\title{
A CORRELATION BETWEEN INCLINATION AND COLOR IN THE CLASSICAL KUIPER BELT
}

\author{
Chadwick A. Trujillo and Michael E. Brown \\ California Institute of Technology, Division of Geological and Planetary Sciences, MS 150-21, Pasadena, CA 91125; \\ chad@gps.caltech.edu, mbrown@caltech.edu \\ Received 2001 October 25; accepted 2002 January 3; published 2002 January 22
}

\begin{abstract}
We have measured broadband optical $B V R$ photometry of 24 classical and scattered Kuiper belt objects (KBOs), approximately doubling the published sample of colors for these classes of objects. We find a statistically significant correlation between object color and inclination in the classical Kuiper belt using our data. The color and inclination correlation increases in significance after the inclusion of additional data points culled from all published works. Apparently, this color and inclination correlation has not been more widely reported because the Plutinos show no such correlation and thus have been a major contaminant in previous samples. The color and inclination correlation excludes simple origins of color diversity, such as the presence of a coloring agent without regard to dynamical effects. Unfortunately, our current knowledge of the Kuiper belt precludes us from understanding whether the color and inclination trend is due to environmental factors, such as collisional resurfacing, or primordial population effects. A perihelion and color correlation is also evident, although this appears to be a spurious correlation induced by sampling bias, as perihelion and inclination are correlated in the observed sample of KBOs.
\end{abstract}

Subject headings: comets: general — Kuiper Belt — Oort Cloud

\section{INTRODUCTION}

Beyond Neptune, the solar system is populated by $\sim 10^{5}$ Kuiper belt objects (KBOs, also trans-Neptunians) larger than $100 \mathrm{~km}$ in diameter, which represent about 0.1 Earth masses of material and are presumed to contain the least thermally processed material in the solar system (Trujillo, Jewitt, \& Luu 2001). As of 2002 January, $\approx 550 \mathrm{KBOs}$ are known, less than $1 \%$ of the inferred population. About $90 \%$ of the observed KBOs can be grouped into one of three dynamical classes: (1) the classical KBOs represent two-thirds of the observed sample and about one-half of the inferred population; (2) the resonant KBOs are comprised primarily of Plutinos $(a \approx 39.4 \mathrm{AU})$ and objects in $2: 1$ mean motion resonance with Neptune $(a \approx 47.7 \mathrm{AU})$, representing $15 \%$ of the observed sample but only $6 \%$ of the total population; and (3) the scattered KBOs comprise about $10 \%$ of the observed sample but nearly half the inferred population because their extreme dynamical properties $(50 \mathrm{AU}<a<$ $200 \mathrm{AU}$ and eccentricities $0.2<e<0.9)$ place them at unobservably great heliocentric distances for large fractions of their orbits (Trujillo, Jewitt, \& Luu 2000).

Since the KBOs are thought to be the least thermally modified bodies in the solar system, they are expected to provide insight into the physical and chemical processes that dominated the primitive solar nebula. Given the fact that the KBOs have been observed for nearly a decade, very little is known about their physical properties, primarily because of their extreme faintness (median $R$ magnitude $m_{R}=23.0$ ). The most striking physical feature observed of the KBOs is their substantial color diversity, from nearly neutral reflectance to the reddest objects in the solar system (Jewitt \& Luu 1998; Tegler \& Romanishin 2000). Objects are reddened from the visible throughout the near-IR (Davies et al. 2000), presumably from a single coloring agent (Jewitt \& Luu 2001), although the physical and chemical mechanisms for the reddening process have not been identified. Recently, Tegler \& Romanishin (2000) reported that the classical KBOs exhibited a correlation between color and inclination. No other observers have reported this trend, and Tegler \& Romanishin estimate the significance of their reported trend using their two-color paradigm, which has not been confirmed by independent sources (Barucci et al. 2001; Jewitt \& Luu 2001). Thus, we chose to conduct our own $B V R$ observations of this population. Using our new observations, we demonstrate that the inclination and color correlation is indeed statistically significant. In addition, we note the presence of a perihelion and color correlation that appears to be due to sampling bias.

\section{OBSERVATIONS}

We chose to observe the classical KBOs-those KBOs that are expected to be the least dynamically modified by Neptune. In practice, 19 classical KBOs and five scattered KBOs were observed because of the distribution of objects in the sky when observations were scheduled. Results of this work are not changed significantly by the inclusion or exclusion of the scattered KBOs since they are a minority population in our sample. All data were taken with the same equipment, the Low Resolution Imaging Spectrograph (LRIS; Oke et al. 1995) and the blue channel of the LRIS (LRIS-B; McCarthy et al. 1998) at the $10 \mathrm{~m}$ Keck I telescope during the nights of 2001 February 26-27. This dichroic-based instrument combination allows the simultaneous use of blue and visible filters (yielding $B-V$ color) or blue and red filters (yielding $B-R$ color), allowing a factor of 2 increase in efficiency over the previous singlecamera system. The efficiency of the LRIS/LRIS-B instrument has permitted us to approximately double the published sample of broadband optical colors for the classical KBOs in just two nights.

Standard stars (Landolt 1992) were imaged at a variety of air masses, and photometric reductions were performed using a two-dimensional point-spread function (PSF) fitting procedure, which does not presuppose a radially symmetric PSF. Using this method, more than 20 bright field stars were sampled in both spatial dimensions of the focal plane to estimate the true PSF for a given image. Since exposure times were fixed at 5 minutes, $\mathrm{KBO}$ apparent motion $\left(3^{\prime \prime} \mathrm{hr}^{-1}\right.$ or 0 ".25 per exposure) was minimized, yielding no significant difference between the KBO PSFs and the stellar PSFs given the $\sim 1^{\prime \prime} B$ band seeing (seeing for the $R$ and $V$ bands was similar since the focus was optimized for $B$ ). The stellar PSF was fitted to 


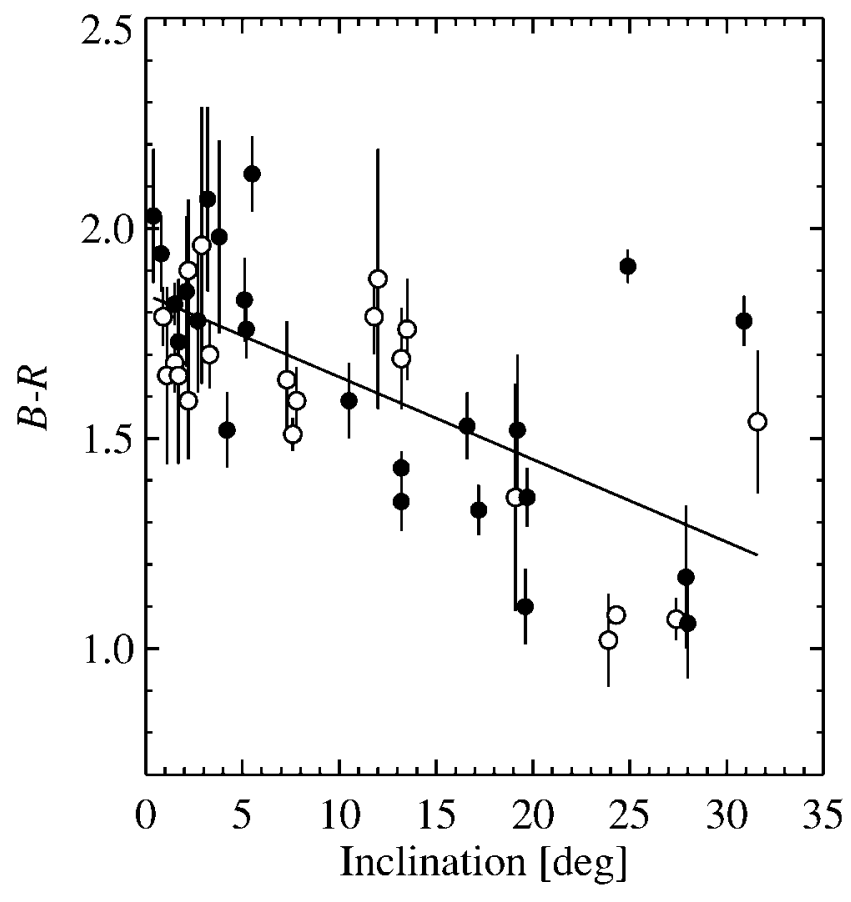

FIG. 1. $-B-R$ color vs. inclination for the classical KBOs and scattered KBOs in our sample (filled circles) and all published data (no Plutinos; open circles). A linear least-squares fit has also been plotted for illustrative purposes (solid line; slope $=-0.0196 \pm 0.003 \mathrm{mag} \mathrm{deg}^{-1}$, intercept $=1.84 \pm 0.5 \mathrm{mag}$ ). The trend corresponds to $3.1 \sigma$ (our data points) and $4.1 \sigma$ (all data points) significance, as estimated by the Spearman rank correlation method.

the KBO image to determine flux and local background levels. In all cases, KBO PSFs were consistent with the model PSF within the Poisson noise. Additionally, synthetic data tests were performed to demonstrate that the PSF fitting procedure is robust to errors in KBO centroiding. Approximately four $B$-band, two $V$-band, and two $R$-band measurements were taken of each $\mathrm{KBO}$, although measurements were repeated if contamination by background sources was suspected. The reduced data appear free of instrumental biases - no correlations were observed between color and data collection parameters such as air mass, seeing, time of observations, apparent motion of objects, location in the focal plane, or Sun-target-observer angle. The most striking feature of these data appears in Figure 1, which shows an apparent correlation between orbital inclination $i$ and $B-R$ color. With few exceptions, this correlation appears to vary continuously from the reddest (low-inclination) KBOs to the bluest (high-inclination) KBOs. Both the $B-R$ and $B-V$ color measurements for each object, corrected to zero air mass, are presented in Table 1. The physical implications of these observations are discussed in $\S 3$, while an estimation of the statistical significance of the color and inclination trend, as well as other trends, appears in the remainder of this section.

\subsection{The KBO Inclination and Color Correlation}

To estimate the significance of the relationship between color and $i$, we computed the Spearman (1904) rank correlation statistic $r$ for our data set, which takes on nonzero values in the range $-1<r<1$ for correlated quantities. The strength of the Spearman method is that it does not presuppose a functional form for the correlation, as do linear least-squares estimates. In addition, the Spearman method is less sensitive to the presence of deviant data points than a $\chi^{2}$ correlation test, as it is the difference in rank between sorted data lists that is compared,
TABLE 1

PHOtOMETRIC DATA From This Work

\begin{tabular}{|c|c|c|c|c|}
\hline Object & $\begin{array}{c}i^{\mathrm{a}} \\
(\mathrm{deg})\end{array}$ & $\begin{array}{c}R \\
(\mathrm{mag})\end{array}$ & $\begin{array}{c}B-R \\
(\mathrm{mag})\end{array}$ & $\begin{array}{c}B-V \\
(\mathrm{mag})\end{array}$ \\
\hline $999 \mathrm{HU}_{11}$ & 0.4 & $23.04 \pm 0.06$ & $2.03 \pm 0.16$ & $1.36 \pm 0.16$ \\
\hline $99 \mathrm{CO}_{153}$ & 0.8 & $22.69 \pm 0.03$ & $1.94 \pm 0.09$ & $1.03 \pm 0.10$ \\
\hline $97 \mathrm{CU}_{29}{ }^{\mathrm{b}}$ & 1.5 & $22.59 \pm 0.03$ & $1.82 \pm 0.05$ & $1.10 \pm 0.05$ \\
\hline $1994 \mathrm{EV}_{3}^{\mathrm{b}}$ & 1.7 & $23.64 \pm 0.06$ & $1.73 \pm 0.15$ & $1.15 \pm 0.23$ \\
\hline $2000 \mathrm{FS}_{53}$ & 2.1 & $23.29 \pm 0.05$ & $1.85 \pm 0.18$ & $1.23 \pm 0.20$ \\
\hline 999 CM & 2.7 & $23.58 \pm 0.06$ & $1.78 \pm 0.17$ & $0.90 \pm 0.19$ \\
\hline $1999 \mathrm{CJ}_{119}$. & 3.2 & $22.92 \pm 0.21$ & $2.07 \pm 0.22$ & $1.38 \pm 0.18$ \\
\hline $2000 \mathrm{CN}$ & 3.8 & $2.46 \pm 0$. & $1.98 \pm 0.23$ & $1.14 \pm 0.23$ \\
\hline $2000 \mathrm{CL}_{105} \ldots \ldots$ & 4.2 & $23.25 \pm 0$ & $1.52 \pm 0.09$ & $1.11 \pm 0.08$ \\
\hline $1999 \mathrm{HT}_{11}$ & 5.1 & $23.05 \pm 0$ & $1.83 \pm 0.10$ & $1.14 \pm 0.14$ \\
\hline $9 \mathrm{GS}_{4}$ & 5.2 & $22.64 \pm 0$ & $1.76 \pm 0.07$ & $1.13 \pm 0.09$ \\
\hline $9 \mathrm{CV}$ & 5.5 & $22.78 \pm 0.06$ & $2.13 \pm 0.09$ & $1.08 \pm 0.08$ \\
\hline JD & 10.5 & $22.26 \pm 0.02$ & $1.59 \pm 0.09$ & $1.11 \pm 0.10$ \\
\hline $1999 \mathrm{CQ}_{133}$ & 13.2 & $23.07 \pm 0.05$ & $1.35 \pm 0.07$ & $0.75 \pm 0.07$ \\
\hline $1999 \mathrm{HB}_{12}$ & 13.2 & $22.12 \pm 0.02$ & $1.43 \pm 0.04$ & $0.88 \pm 0.06$ \\
\hline $1999 \mathrm{CG}_{\text {. }}$ & 16.6 & $23.21 \pm 0.04$ & $1.53 \pm 0.08$ & $0.87 \pm 0.09$ \\
\hline $1999 \mathrm{HV}$ & 17.2 & $22.93 \pm 0.03$ & $1.33 \pm 0.06$ & $0.80 \pm 0.08$ \\
\hline $2000 \mathrm{CO}_{105}$ & 19.2 & $22.58 \pm 0.18$ & $1.52 \pm 0.18$ & $0.82 \pm 0.06$ \\
\hline $2000 \mathrm{CQ}_{105} \ldots \ldots$ & 19.6 & $23.02 \pm 0.05$ & $1.10 \pm 0.09$ & $0.71 \pm 0.09$ \\
\hline $1999 \mathrm{CF}_{119} \ldots \ldots \ldots$ & 19.7 & $22.90 \pm 0.04$ & $1.36 \pm 0.07$ & $0.77 \pm 0.07$ \\
\hline $1999 \mathrm{KR}$ & 24.9 & $21.31 \pm 0.03$ & $1.91 \pm 0.04$ & $1.13 \pm 0.06$ \\
\hline & 27.9 & $23.40 \pm 0$ & $1.17 \pm 0.17$ & $0.78 \pm 0.09$ \\
\hline $1998 \mathrm{HL}_{151}$ & 28.0 & $23.96 \pm 0.08$ & $1.06 \pm 0.13$ & $0.59 \pm 0.19$ \\
\hline $2000 \mathrm{AF}_{255} \ldots \ldots$ & 30.9 & $22.92 \pm 0.03$ & $1.78 \pm 0.06$ & $1.16 \pm 0.07$ \\
\hline
\end{tabular}

NoTE. - Objects are ordered by increasing inclination. Orbital elements were provided by the Minor Planet Center.

${ }^{\mathrm{a}}$ Inclination with respect to the ecliptic. Transforming the inclination to the invariable plane (mean correction 0.6; Burkhardt 1982) does not have a significant impact on results. Semimajor axis.

${ }^{\mathrm{b}}$ These objects were also measured by another group (see Table 2).

not the actual data values themselves. Two statistical tests (Press et al. 1992, pp. 640-641), the $t$-statistic and the variance of the sum squared difference of ranks, $\operatorname{Var}(D)$, can be used to estimate the significance of a nonzero value of $r$ (here we report the mean of the two tests). We measure the correlation between $B-R$ color (a stronger indicator of color than $B-V$ ) and orbital inclination as $r=-0.62$ for the $N=24$ data points in our new sample. The probability of this $r$ or a more significant one occurring in an uncorrelated sample is $P=2.0 \times$ $10^{-3}$ (3.1 $\sigma$ significance, assuming Gaussian statistics, since $1-P$ yields the confidence level).

In order to increase sample size, we repeated this analysis, combining our data with all previously published data (Table 2). Color measurements of the same object from multiple investigators have been combined into one value, with error bars reflecting the greater of (1) the standard error of the color values or (2) the mean of the reported errors when combined in quadrature. We note that the process that produces the colors appears stochastic in nature, with individual KBOs departing from the color correlation based on their past interactions with the coloring mechanism. Error bars reflect color measurement error and not the expected departure of an individual KBO from the overall color/inclination trend, which cannot be computed a priori without more information about the physical nature of the coloring process. We note that in cases of duplication between our objects and published objects, our color values are statistically indistinguishable from other published values. By increasing the sample size using previously published data on all KBOs, the significance of the inclination and color correlation increases to $3.3 \sigma$ $(N=60)$.

It appears that the correlation between color and inclination has not been widely reported because of the influence of the Plutinos, for which no correlation is apparent. Removing the 
TABLE 2

Photometric Data from All Other Published Works

\begin{tabular}{|c|c|c|c|c|}
\hline Object & $\begin{array}{c}i^{\mathrm{a}} \\
(\mathrm{deg})\end{array}$ & $\begin{array}{c}B-R \\
(\mathrm{mag})\end{array}$ & $\begin{array}{c}B-V \\
(\mathrm{mag})\end{array}$ & References \\
\hline $1998 \mathrm{WX}_{24}$ & 0.9 & $1.79 \pm 0.07$ & $1.09 \pm 0.05$ & 1 \\
\hline $1994 \mathrm{ES}_{2} \ldots \ldots \ldots$ & 1.1 & $1.65 \pm 0.21$ & $0.71 \pm 0.15$ & 2 \\
\hline $1994 \mathrm{VK}_{8} \ldots \ldots \ldots$ & 1.5 & $1.68 \pm 0.07$ & $1.01 \pm 0.06$ & 1 \\
\hline $1997 \mathrm{CU}_{29} \ldots \ldots \ldots$ & 1.5 & $2.19 \pm 0.48$ & $1.22 \pm 0.10$ & 3,4 \\
\hline $1994 \mathrm{EV}_{3} \ldots \ldots \ldots$ & 1.7 & $2.04 \pm 0.20$ & $1.50 \pm 0.19$ & 2 \\
\hline $1995 \mathrm{WY}_{2} \ldots \ldots \ldots$ & 1.7 & $1.65 \pm 0.21$ & $1.01 \pm 0.16$ & 2,4 \\
\hline $1992 \mathrm{QB}_{1} \ldots \ldots \ldots$ & 2.2 & $1.59 \pm 0.14$ & $0.85 \pm 0.10$ & $1,2,4$ \\
\hline $1997 \mathrm{CS}_{29} \ldots \ldots \ldots$ & 2.2 & $1.90 \pm 0.17$ & $1.10 \pm 0.04$ & $3,4,5$ \\
\hline $1997 \mathrm{CQ}_{29} \ldots \ldots$ & 2.9 & $1.96 \pm 0.33$ & $0.99 \pm 0.09$ & 3,4 \\
\hline $1996 \mathrm{TK}_{66} \ldots \ldots \ldots$ & 3.3 & $1.70 \pm 0.08$ & $1.04 \pm 0.07$ & 1,4 \\
\hline $1996 \mathrm{TS}_{66} \ldots \ldots \ldots$ & 7.3 & $1.64 \pm 0.14$ & $1.02 \pm 0.05$ & $4,5,6$ \\
\hline $1999 \mathrm{DE}_{9} \ldots \ldots \ldots$ & 7.6 & $1.51 \pm 0.04$ & $0.94 \pm 0.03$ & 4 \\
\hline 1993 FW …..... & 7.8 & $1.59 \pm 0.08$ & $0.95 \pm 0.06$ & 2,3 \\
\hline $1997 \mathrm{SZ}_{10} \ldots \ldots$ & 11.8 & $1.79 \pm 0.09$ & $1.14 \pm 0.08$ & 1 \\
\hline $1998 \mathrm{WH}_{24} \ldots \ldots$ & 12.0 & $1.88 \pm 0.31$ & $0.94 \pm 0.04$ & 1,3 \\
\hline $1997 \mathrm{QH}_{4} \ldots \ldots \ldots$ & 13.2 & $1.69 \pm 0.12$ & $1.03 \pm 0.10$ & 1,4 \\
\hline $1998 \mathrm{SM}_{165} \ldots \ldots$ & 13.5 & $1.76 \pm 0.12$ & $1.01 \pm 0.10$ & 1 \\
\hline $1997 \mathrm{CR}_{29} \ldots \ldots$ & 19.1 & $1.36 \pm 0.27$ & $0.67 \pm 0.20$ & 4 \\
\hline $1996 \mathrm{TL}_{66}$. & 23.9 & $1.02 \pm 0.11$ & $0.70 \pm 0.04$ & $4,5,6,7$ \\
\hline $1999 \mathrm{OY}_{3}$. & 24.3 & $1.08 \pm 0.02$ & $0.71 \pm 0.01$ & 1 \\
\hline $1999 \mathrm{KR}_{16} \ldots \ldots$ & 24.9 & $1.84 \pm 0.06$ & $1.10 \pm 0.05$ & 4 \\
\hline $1996 \mathrm{TO}_{66} \ldots \ldots \ldots$ & 27.4 & $1.07 \pm 0.05$ & $0.69 \pm 0.03$ & $4,5,6,7$ \\
\hline $1996 \mathrm{RQ}_{20} \ldots \ldots$ & 31.6 & $1.54 \pm 0.17$ & $0.96 \pm 0.13$ & 7 \\
\hline
\end{tabular}

NoTE. - Objects are presented in order of increasing inclination. Because of space considerations, Plutinos have been excluded from this table. Orbital elements were provided by the Minor Planet Center.

${ }^{\text {a }}$ Inclination with respect to the ecliptic. Transforming the inclination to the invariable plane (mean correction 0.6; Burkhardt 1982) does not have a significant impact on results.

REFERENCES. - (1) Tegler \& Romanishin 2000; (2) Luu \& Jewitt 1996; (3) Barucci et al. 2000; (4) Jewitt \& Luu 2001; (5) Tegler \& Romanishin 1998; (6) Jewitt \& Luu 1998; (7) Barucci et al. 1999.

Plutinos from the combined sample of data results in a large increase in the correlation statistic to $4.1 \sigma(N=44)$. However, we must note that the removal of the Plutinos is still statistically tentative since a two-dimensional Kolmogorov-Smirnov test (Press et al. 1992, pp. 645-649) shows that the null hypothesis that the Plutino and other $\mathrm{KBO}(i, B-R)$-values are drawn from the same distribution can be rejected with only $93 \%$ $(<2 \sigma)$ confidence. This observation in itself has some potential implications for the origin of color diversity, as discussed in the next section. Since the correlation between inclination and color for the non-Plutino KBOs (1) increases in significance with increased sample size, (2) does not appear to be the product of any observational biases, (3) is observed in both $B-V$ and $B-R$, and (4) has a less than $4 \times 10^{-5}(4.1 \sigma)$ chance of being produced in an uncorrelated population, we conclude that it is statistically significant.

\subsection{Other Correlations}

The only other color trend that we observe for the classical $\mathrm{KBOs}$ is a perihelion and color correlation. This trend, however, is almost certainly due to sampling bias. To illustrate this, we have considered two subsamples of the $B-R$ data, which are shown as the gray bars in Figure 2. The first subsample (the "constant $q$ " subsample) shows little perihelion variation but large inclination variation and consists of objects in the range $37 \mathrm{AU}<q<40 \mathrm{AU}$. The constant $q$ subsample exhibits a $2.8 \sigma$ correlation between inclination and color, indicating that the inclination and color correlation cannot be attributed to perihelion variations alone (Fig. 3). The second subsample (the "constant $i$ " subsample) shows little inclination variation but large perihelion variation and consists of objects in the range

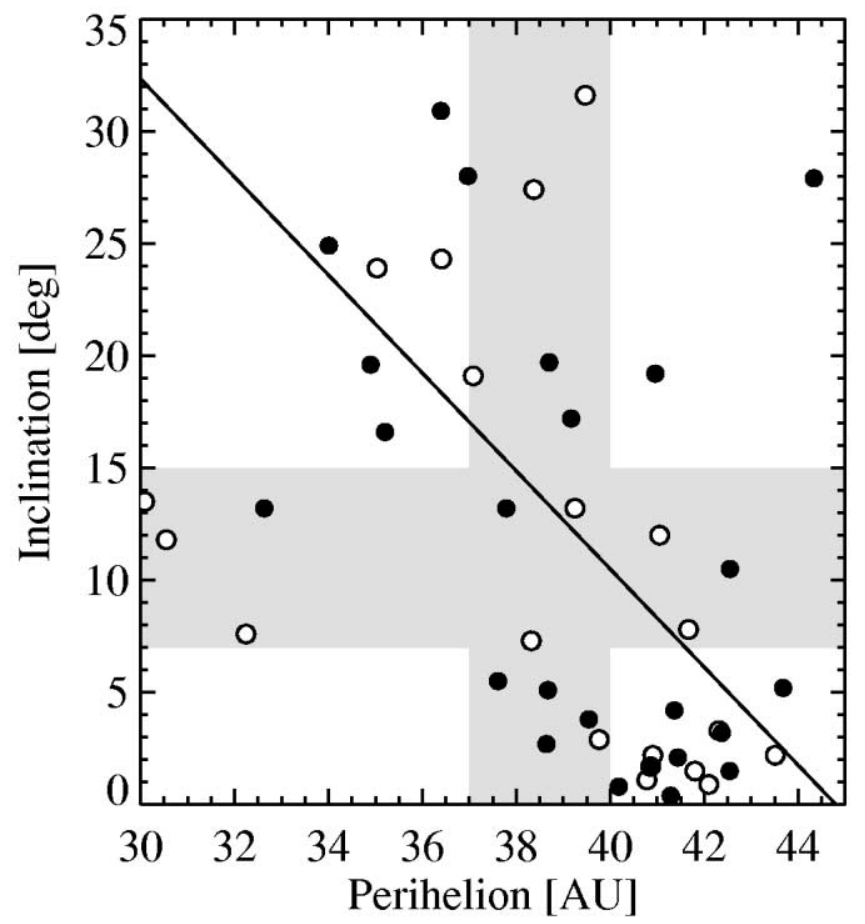

FIG. 2.-Inclination vs. perihelion for the classical KBOs and scattered KBOs in our sample (filled circles) and all published data (no Plutinos; open circles). For illustrative purposes, a linear least-squares fit has been plotted for points with perihelion greater than $34 \mathrm{AU}$ (solid line). The gray bars represent the constant $q$ (vertical bar) and constant $i$ (horizontal bar) subsamples detailed in Fig. 3.

$7^{\circ}<i<15^{\circ}$. The constant $i$ subsample shows no correlation between perihelion and color, indicating that the perihelion and color trend is most likely a spurious correlation induced by sampling bias.

\section{INTERPRETATION}

The link between color and inclination rules out very simple mechanisms of producing the observed KBO color diversity. The presence of a coloring agent (surface frosts, for example) independent of the Kuiper belt dynamical environment cannot be the only cause of color diversity because, in such a scenario, no correlation between color and any orbital parameter would be expected. More complex processes must be invoked such as evolutionary processes or remnant primordial composition effects.

Collisional resurfacing has been suggested as a possible mechanism for producing the wide range of KBO colors (Luu \& Jewitt 1996; Jewitt \& Luu 2001). In this model, a close competition between radiative processing, which reddens the surface, and collisional resurfacing, which restores neutral ices to the surface, produces the observed color variations. Assuming that collisions are most likely to take place near the ecliptic where the KBO number densities are highest, a color and inclination correlation seems likely. An object's speed perpendicular to the ecliptic follows $\Delta v \propto \sin i$. From laboratory simulations (Fujiwara, Kamimoto, \& Tsukamoto 1977) scaled to solar system events (Fujiwara et al. 1989), the mass ejected during a collision is proportional to impact energy squared, $m \propto E^{2} \propto \Delta v^{4}$. Thus, ejecta mass could be a strong function of object inclination, $m \propto$ $\sin ^{4} i$, leading to a correlation between color and inclination. If this were the case, one might expect to see a correlation between color and population-averaged impact velocity $v_{\text {imp }} \propto\left(i^{2}+\right.$ 

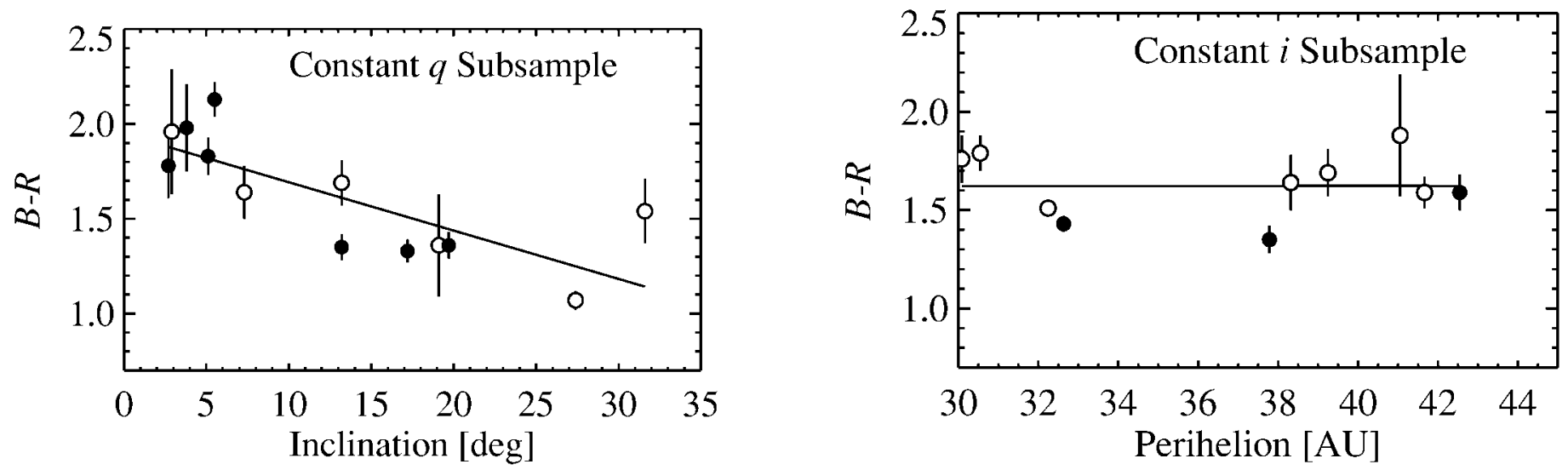

FIG. 3.-Color vs. inclination for the constant $q$ subsample (left) and color vs. perihelion for the constant $i$ subsample (right). The left panel corresponds to 2.8 $\sigma$, and the right panel is not significant, as estimated by the Spearman rank correlation method. The constant perihelion subsample (left) indicates that the inclination/ color correlation is not due to observational bias. In contrast, the constant $i$ subsample lacks any trend, indicating that the observed perihelion/color correlation is most likely due to sampling bias induced by the perihelion/inclination correlation.

$\left.e^{2}\right)^{1 / 2}$ (Stern 1995). Unfortunately, because of the paucity of color data, it is not currently possible to separate the effects of an $i$ correlation from a $v_{\text {imp }}$ correlation because the two parameters are so strongly correlated. In addition, it is not clear why the collisional environment would be different for the Plutinos, which show no correlations between $i$ and color. Further modeling is required to fully quantify this effect. Unfortunately, the competition between collisions and radiative processing is quite difficult to model since we do not have a thorough understanding of all of the astrophysical effects that take place. For instance, no dynamical models have yet estimated collision rates based on population dynamics in a manner that could demonstrate a dynamics-induced color correlation. In addition, models of radiative processing have not addressed the effect of the complete carbonization of surfaces, which would result in a dark, neutrally colored surface. Furthermore, no collisional resurfacing models have adequately reproduced the magnitude of color variations presently observed for the KBOs (Jewitt \& Luu 2001).

The best alternative to evolutionary processes is that the colors are primordial in nature. An $i$ correlation might be ex- pected if the KBOs were composed of multiple subpopulations with different primordial colors and inclinations. Such a model is compatible with two recently reported dynamical groupings: (1) the Levison \& Stern (2001) large- and small-size components of the classical KBOs and (2) the Brown (2001) high- $i$ and low- $i$ components of the classical KBOs. However, we find no correlations between color and any orbital parameter other than $i$, as might be expected for a cosmogonic origin to the colors. Even if the KBO colors are dominated by primordial processes, the role of collisions in altering KBO surfaces must still be addressed given that (1) collisions are thought to be responsible for reducing the mass of the Kuiper belt by a factor of $\sim 100$ since the epoch of formation (Stern \& Colwell 1997; Kenyon \& Luu 1999) and (2) it is expected that a large fraction of KBO surfaces are covered with impact signatures (Durda \& Stern 2000).

Antonin Bouchez provided observational assistance at the telescope. David Sprayberry and Gabrelle Saurage provided technical and operational assistance at the telescope.

\section{REFERENCES}

Barucci, M. A., Doressoundiram, A., Tholen, D., Fulchignoni, M., \& Lazzarin, M. 1999, Icarus, 142, 476

Barucci, M. A., Fulchignoni, M., Birlan, M., Doressoundiram, A., Romon, J., \& Boehnhardt, H. 2001, A\&A, 371, 1150

Barucci, M. A., Romon, J., Doressoundiram, A., \& Tholen, D. J. 2000, AJ, 120,496

Brown, M. E. 2001, AJ, 121, 2804

Burkhardt, G. 1982, A\&A, 106, 133

Davies, J. K., Green, S., McBride, N., Muzzerall, E., Tholen, D. J., Whiteley,

R. J., Foster, M. J., \& Hillier, J. K. 2000, Icarus, 146, 253

Durda, D. D., \& Stern, S. A. 2000, Icarus, 145,220

Fujiwara, A., Kamimoto, G., \& Tsukamoto, A. 1977, Icarus, 31, 277

Fujiwara, A., et al. 1989, in Asteroids II, ed. R. P. Binzel, T. Gehrels, \& M. S. Matthews (Tucson: Univ. Arizona Press), 240

Jewitt, D., \& Luu, J. 1998, AJ, 115, 1667

. 2001, AJ, 122, 2099
Kenyon, S. J., \& Luu, J. X. 1999, AJ, 118, 1101

Landolt, A. U. 1992, AJ, 104, 340

Levison, H. F., \& Stern, S. A. 2001, AJ, 121, 1730

Luu, J., \& Jewitt, D. 1996, AJ, 112, 2310

McCarthy, J. K., et al. 1998, Proc. SPIE, 3355, 81

Oke, J. B., et al. 1995, PASP, 107, 375

Press, W. H., Teukolsky, S. A., Vetterling, W. T., \& Flannery, B. P. 1992, Numerical Recipes in C: The Art of Scientific Computing (2d ed.; Cambridge: Cambridge Univ. Press)

Spearman, C. 1904, Am. J. Psychology, 15, 72

Stern, S. A. 1995, AJ, 110, 856

Stern, S. A., \& Colwell, J. E. 1997, ApJ, 490, 879

Tegler, S. C., \& Romanishin, W. 1998, Nature, 392, 49

2000, Nature, 407, 979

Trujillo, C. A., Jewitt, D. C., \& Luu, J. X. 2000, ApJ, 529, L103

. 2001, AJ, 122, 457 